\title{
HABITAT SELECTION UNDER TEMPORAL HETEROGENEITY: EXORCIZING THE GHOST OF COMPETITION PAST
}

\author{
Kenneth A. Schmidt, ${ }^{1,5}$ Joanne M. Earnhardt, ${ }^{2,3}$ Joel S. Brown, ${ }^{2}$ And Robert D. Holt ${ }^{4}$ \\ ${ }^{1}$ Institute of Ecosystem Studies, Box AB, Millbrook, New York 12545 USA \\ ${ }^{2}$ Department of Biological Sciences, University of Illinois, 845 W. Taylor, Chicago, Illinois 60607 USA \\ ${ }^{3}$ Lincoln Park Zoo, 2001 N. Clark St., Chicago, Illinois 60614 USA \\ ${ }^{4}$ Museum of Natural History, University of Kansas, Lawrence, Kansas 66045 USA
}

\begin{abstract}
We investigate how coexistence between competitors may be influenced by habitat selection when habitats represent either sources or sinks, and given that dispersal is free to evolve. Evolutionary stable dispersal between source and sink habitats can occur if local fitnesses vary temporally, either due to intrinsic factors (e.g., chaotic dynamics) or extrinsic factors (e.g., environmental stochasticity). The model assumes locally linear LotkaVolterra competition between two species. Given sufficiently low density-independent mortality in the sink, dispersal between habitats is an evolutionary stable strategy (ESS). Given a trade-off between competitive ability in the source and mortality in the sink, a sink habitat can promote species coexistence in the source habitat if the inferior competitor species experiences lower mortality in the sink. This highlights how sink habitats may provide mechanisms of coexistence in heterogeneous landscapes. In a second scenario, the competitors have distinct habitat preferences, resulting in the "Ghost of Competition Past" (i.e., complete habitat partitioning) with stable population dynamics. With unstable population dynamics, dispersal between habitats becomes the ESS, and the Ghost vanishes leading either to coexistence of the competitors in both habitats or global exclusion of one species. Our results highlight the importance of jointly considering the effects of spatial heterogeneity and temporal variability when analyzing the coexistence of competing, mobile organisms.
\end{abstract}

Key words: evolutionary stable strategy (ESS); Ghost of Competition Past; habitat selection; mechanisms of coexistence; source-sink dynamics; spatial stochasticity; temporal stochasticity.

\section{INTRODUCTION}

A central theme in community ecology is that the species diversity of communities reflects the spatial and temporal heterogeneity of environments (Chesson and Rosenzweig 1991, Rosenzweig 1991, Brown 1996, Hairston et al. 1997). Individual behavior helps define how individuals experience such heterogeneity, and thus determines the strength and character of interspecific interactions. Habitat selection by individuals in particular can strongly influence the likelihood of coexistence between competitors (e.g., see reviews by Rosenzweig 1991, Brown 1997). For instance, consider the model with distinct habitat preferences developed by Rosenzweig (1991). Each of two or more species is assumed to exploit its preferred habitat more efficiently than does any competing species. If a given species is alone, then as its density rises in its preferred habitat, there is a threshold density at which the species begins to use its less preferred habitat(s). When competing species are present, increases in the density of the competing species in the less preferred habitat in-

Manuscript received 23 December 1998; revised 22 July 1999; accepted 12 August 1999

${ }^{5}$ Present address: University of Memphis, Department of Biology, 3700 Walker Avenue, Memphis, Tennessee 38152 USA. E-mail: thatseasy@aol.com crease this threshold for habitat generalization. If the threshold density in the preferred habitat at which a species begins using its less preferred habitats exceeds its equilibrium density in its preferred habitat, it will be restricted to its preferred habitat. Moreover, small changes in the density of either species at equilibrium do not induce a shift in either the abundance or habitat distribution of the other species. The general result is that given ideal-free habitat selection, distinct habitat preferences, and equilibrium conditions, each species is restricted to a single habitat and does not respond to small perturbations in habitat use or abundance. This phenomenon of habitat partitioning arising from habitat selection has been termed the "Ghost of Competition Past" (Rosenzweig 1981, 1987). A number of papers (Brew 1982, Parker and Sutherland 1986, Brown 1990, 1996) have examined the input of habitat selection upon competitive coexistence in a spatially heterogeneous landscape, assuming that systems tend towards a stable equilibrium, with broadly comparable conclusions.

The predictions of a "ghost" phenomenon in a spatially heterogeneous landscape assume that the system settles into an equilibrium, with all species persisting at constant abundances. But natural communities typically exhibit nonequilibrial abundances where abundances may vary by orders of magnitude (Connell and 
Sousa 1983, Fryxell et al. 1998). In this paper, we reexamine the influence of habitat selection upon competitive coexistence in spatially heterogeneous landscapes, but relax the assumption of equilibrial dynamics. Moreover, we will permit one of the habitats to be a population sink for one or more competing species (Shmida and Ellner 1984, Pulliam 1988), wherein immigration balances local population decline. In the simplest case, a sink habitat is a "hard" sink, where fitness is less than unity at all densities, even in the absence of interspecific competition. A habitat may also be a "soft" sink because of sufficiently abundant conspecific or heterospecific competitors (somewhat similar to the "pseudosinks" discussed by Watkinson and Sutherland 1995).

The influence of sink habitats on competitive coexistence has not been previously examined. Given optimal habitat selection and demographic equilibrium, dispersal into a sink habitat appears to be evolutionarily unstable, in that individuals are moving down gradients in fitness (MacArthur 1972, Holt 1985). Today, we recognize at least three general reasons why dispersal to a sink may be evolutionarily stable (Holt 1997), provided descendants can back-immigrate to the source (Morris 1991). First, interference or pre-emption of territories or breeding sites in high-quality habitats (Pulliam 1988, Pulliam and Danielson 1991) may make it advantageous for some individuals to occupy a sink habitat, despite an absolute fitness there of less than one. Second, constraints upon movement or dispersal may lead to distributions that are nonideal (e.g., see Keddy 1981). Third, sinks may persist within the habitat repertoire of a species due to unstable population dynamics in source habitats (Holt 1997). Unstable dynamics necessarily implies temporal variance in fitness, with both good and poor years. Dispersal to a sink habitat may hedge an individual's bets in the face of unpredictability in the source; in effect, sinks may store recruitment from source habitats during good years, buffering excursions to low fitness in bad years (Holt 1997, Vincent et al. 1998).

Temporal variability should have a profound influence on a species' distribution across habitats. If multiple species are present and competing, it is likely that temporal variability will also influence the development of habitat partitioning among competitors. In the models discussed below, we assume that the ability to persist within sinks comes at the expense of competitive ability in the source (i.e., a trade-off between mortality in the sink and competitive ability in the source). We will show that the presence of sink habitats can modify or even reverse patterns of species coexistence at the local scale as well as the entire landscape.

A competing species may make a habitat a soft sink, reducing population growth rate in the focal species below replacement there. Indeed, the "Ghost of Competition Past" scenario works through the creation of soft sinks which are avoided at equilibrium because of optimal habitat selection. Analogous to the influence of temporal variability on the evolutionarily stable use of hard sinks, temporal variability could tend to promote the use of soft sinks, despite the presence of competitors (Wilson and Yoshimura 1994, Fryxell 1997). This should lead to a breakdown of the Ghost scenario, as one or more species expands its habitat breadth. Thus, a powerful mechanism of species coexistence and an evolutionary source of diversity arising from habitat selection (Rosenzweig 1987) may not operate as effectively given temporally variable habitats. However, because temporal variability also fosters the use of hard sink habitats, if inferior competitors are differentially able to utilize the sink, the interplay of temporal and spatial heterogeneity may also provide a novel mechanism of coexistence, when neither alone suffices. The models presented below display both these effects.

Evolutionary stable use of sink habitats has previously been modeled as a single-species evolutionary game given intrinsic temporal variability due to limit cycles (Holt 1997) and chaos (Doebeli and Ruxton 1997, see also Holt and McPeek 1996). We focus on the impact of extrinsic temporal variability due to environmental stochasticity (also see Wilson and Yoshimura 1994, Vincent et al. 1998). We examine dispersal as an evolutionary stable strategy (ESS) in each of two species competing in accord with a discrete-time version of the Lotka-Volterra competition equations. Our goal is to illustrate broad implications of dispersal into sinks as factors modifying the coexistence of two competing species, rather than to present an exhaustive examination of the detailed dynamics of the system.

\section{THE ModeL}

We begin by considering a single species. Following Holt (1997), assume that in generation $t$ there are $N(\mathrm{t})$ individuals, a fraction, $p$, of whom settle into habitat A; the remaining $(1-p)$ settle into habitat B. Habitat selection is cost free, and $p$ gives the proportion of individuals within habitat A. Dispersal is unconditional given $p=0.5$, but otherwise combines a preference for one or the other habitat.

To generalize the model to any number of dispersal strategies, let $F_{A}[\mathbf{N}(t), \mathbf{p}]$ and $F_{B}[\mathbf{N}(t), \mathbf{p}]$ be the fitnesses in habitats $\mathrm{A}$ and $\mathrm{B}$, respectively, where $\mathbf{p}=\left(p_{1}, \ldots\right.$, $\left.p_{n}\right)$ is the vector of heritable strategies $\left(0 \leq p_{i} \leq 1\right)$, and $\mathbf{N}(t)=\left(N_{1}, \ldots, N_{n}\right)$ is the vector of the strategies' population sizes. We use a fitness-generating function (Vincent and Brown 1984) to characterize the fitness of all individuals regardless of strategy. Fitness, $G$, for a temporally variable habitat, is best represented by the long-term geometric mean growth rate:

$$
\begin{aligned}
G(p, \mathbf{p}, \mathbf{N})=\prod & \left\{p F_{A}[\mathbf{N}(t), \mathbf{p}]\right. \\
& \left.+(1-p) F_{B}[\mathbf{N}(t), \mathbf{p}]\right\}^{q_{k}}
\end{aligned}
$$

where $q_{k}$ represents the probability that the $k$ th year 
type occurs. In a persisting population, long-term geometric mean fitness is unity. The fitness of an individual using strategy $p_{i}$ can be found by replacing $p$ in Eq. 1 with $p_{i}$ (Vincent and Brown 1984). As an evolutionary stable strategy (ESS), $p_{i}{ }^{*}$ must maximize fitness when all other individuals in the population also use strategy $p_{i}{ }^{*}$. This is determined if $p_{i}^{*}$ satisfies the first and second order necessary conditions for the ESS, $\partial G / \partial p_{i}=$ 0 , and $\partial G^{2} / \partial\left(p_{i}\right)^{2}<0$, respectively, evaluated at $p_{i}=$ p (Vincent and Brown 1984). Solving the first condition and rearranging leads to the following ESS condition (for simplicity, we let $F_{i}=F_{i}[N(t), p]$, where $i=A, B$ ):

$$
\partial G /\left.\partial p_{i}\right|_{p_{i}=\mathbf{p}}=\sum_{k} \frac{q_{k}\left(F_{A}-F_{B}\right)}{\left[p F_{A}+(1-p) F_{B}\right]}=0 .
$$

Algebraic manipulation shows that the second ESS condition holds. To get an explicit solution for the ESS, $p_{i}{ }^{*}$, one must have explicit fitness functions $F_{A}, F_{B}$.

The ESS condition for the two-species model is similar to Eq. 2. Each species has a fitness-generating function, and fitnesses in the habitats are determined jointly by the population density and strategy of both competing species. Solving for the ESS requires simultaneously satisfying the necessary conditions for each species' fitness-generating function. Because in general one cannot solve for density, it is difficult to characterize the ESS analytically. Here we report extensive numerical studies.

\section{Ecological dynamics}

Model 1: hard sink.-The first model contains a source habitat (habitat A) where populations of each species experience competition and density dependent growth, given by $F_{A}[\mathbf{N}(t), \mathbf{p}]$, and a sink habitat (habitat B) where populations of each species experience density independent mortality, $F_{B}<1$. We use a discrete version of the Lotka-Volterra competition equations to describe population growth in the source habitat. This leads to the following growth equations for species $i$ (with competitor $j$ ):

Source:

$$
F_{i A}=\left(1+r_{i A}\left[K_{i A}-\mathbf{p}_{i} \mathbf{N}_{i}(t)-\alpha_{i j} \mathbf{p}_{j} N_{j}(t)\right] / K_{i A}\right)
$$

Sink:

$$
F_{i \mathrm{~B}}=\left(1+r_{i B}\right)
$$

with $-1<r_{i B}<0$, where $r_{i A}$ and $r_{i B}$ are the species' intrinsic growth (or mortality) rates for habitats A and B respectively, $K_{i A}$ is the carrying capacity in habitat $\mathrm{A}$, and $\alpha_{i j}$ is the interaction coefficient of species $j$ on species $i$. Substituting Eqs. 3 into Eq. 1 and finding the solution that satisfies Eq. 2 (for each species simultaneously) in principle determines the ESS. Once the ESS is obtained we can evaluate species' densities and the ESS to examine species coexistence at the local and landscape level. This model illustrates one example of how temporal variability can foster species coexistence, by permitting the use of low-quality sink habitat.

Model 2: soft sink.-In the second model, we envision density dependent growth and positive carrying capacities in each habitat. We consider these habitats as soft sinks, because in the absence of the competing species, either species can persist in either habitat. However, the presence of both species together causes competitive exclusion of each competitor from its less preferred habitat when the system is at equilibrium (i.e., evolutionarily stable habitat partitioning). As above, we used the discrete-time version of the Lotka-Volterra equations to model population growth in the two habitats:

$$
\begin{gathered}
F_{i A}=\left(1+r_{i A}\left[K_{i A}-\mathbf{p}_{i} \mathbf{N}_{i}(t)-\alpha_{i j} \mathbf{p}_{j} \mathbf{N}_{j}(t)\right] / K_{i A}\right) \\
F_{i B}=\left(1+r_{i B}\left[K_{i B}-\left(1-\mathbf{p}_{i}\right) \mathbf{N}_{i}(t)\right.\right. \\
\left.\left.-\alpha_{i j}\left(1-\mathbf{p}_{j}\right) \mathbf{N}_{j}(t)\right] / K_{i B}\right) .
\end{gathered}
$$

The Ghost of Competition Past requires nonintersecting zero-growth isoclines for each habitat, such that each species is the superior competitor in a different habitat:

$$
\begin{array}{ll}
K_{1 A}>K_{2 A} / \alpha_{21} & K_{1 A} / \alpha_{12}>K_{2 A} \\
K_{2 B}>K_{1 B} / \alpha_{12} & K_{2 B} / \alpha_{21}>K_{1 B} .
\end{array}
$$

We assume these inequalities hold and that the interaction coefficients are constant across habitat types. This model illustrates how temporal variability can preclude competitive coexistence by breaking down behaviorally mediated habitat partitioning.

\section{SIMULATIONS}

We simulated environmental stochasticity by multiplying carrying capacities, $K$, by a normally distributed random variable with a mean of unity and a standard deviation $(\sigma)$ that varied among runs between 0 and 0.60. Both species experienced the same level of stochasticity within a year and between habitats (model $2)$. Intrinsic growth rates were chosen to preclude intrinsic cycles or chaos (i.e., $r \leq 1.5$ ). To avoid nonnegative carrying capacities at high stochasticity we set a lower limit to our multiplier such that $K$ never fell below $1 \%$ of its mean value (i.e., in general the minimum $K \geq 10$ ). Likewise we set a minimum fitness floor as $F_{A, B} \geq 0.01$, i.e., a habitat's population could not fall below $1 \%$ of its density from the previous year. This truncation was necessary to avoid species becoming extinct as the result of a single or string of bad years while still maintaining high levels of temporal variability (e.g., $K$ fluctuates over two orders of magnitude between years). Thus, we avoided the result that species may become extinct due to very high levels of stochasticity per se, and focused instead on the influence of temporal variability on competitive exclusion.

We ran the model for 2000 (model 1) and 1200 (model 2) generations (for model 2 fewer generations were 


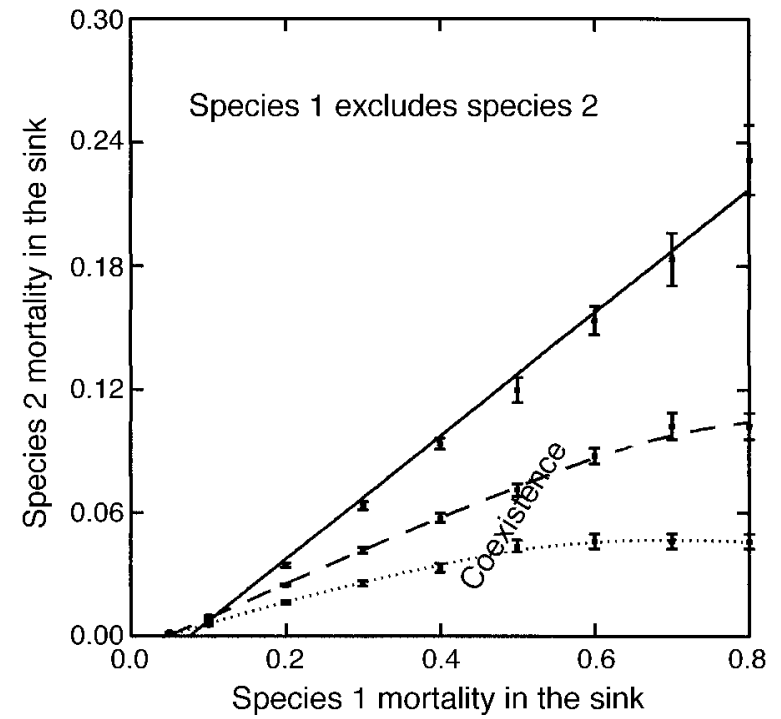

FIG. 1. Regions of coexistence (within the source habitat) and exclusion of the competitive inferior (species 2) plotted in the state space of the mortality of species 1 vs. species 2 in the sink. Species are identical except for competitive ability $\left(\alpha_{12}=0.8, \alpha_{21}=1.1\right)$ and mortality in the sink. Given sufficiently low and high mortality for species 1 and 2, respectively, coexistence within the source habitat occurs. The region of coexistence expands with an increase in the variability of population growth rates in the source $\left(r_{A}=1.0\right.$, solid line; $r_{A}=1.2$, dashed line; $r_{A}=1.5$, dotted line). $K_{1 A}=K_{1 B}=1000$; $\alpha_{12}=0.8 ; \alpha_{21}=1.1 ; \sigma=0.4$.

required to achieve the coexistence or exclusion criteria). In each simulation run, populations were initially seeded at 100 individuals in each habitat. Fitnesses, using Eqs. 3a and b and 4 for model 1 and 2, respectively, and the quotient $\left[q_{k}\left(F_{A}-F_{B}\right)\right] /\left[p F_{A}+(1-\right.$ p) $F_{B}$ ], from Eq. 2, were calculated for each generation. We summed the above quotient over all but the first 200 generations (to allow attainment of a quasi-equilibrium), and varied $p$ in the model until we arrived at the ESS condition as determined by the solution to Eq. 2. After attainment of the ESS solution, we examined the community for local or global extinction and coexistence in one or both habitats. We ran 10 simulations for each combination of parameter values we investigated and report values as the mean $( \pm 1 \mathrm{SE}$ ) for the 10 runs.

We used model 1 to examine the influence on competitive exclusion of a trade-off between competitive ability in the source (habitat A) and density independent mortality in the sink habitat (habitat B). The superior competitor in the source habitat had the higher mortality rate in the sink, but otherwise the species were identical. We set species 1's (superior competitor) mortality in the sink $\left(r_{1 B}\right)$ and varied $r_{2 B}$ until either species existed alone in the source habitat while at the ESS as determined by the methods indicated above (see Fig. 1). We assumed a species went extinct if its mean density over the last 100 generations fell below 1 . This

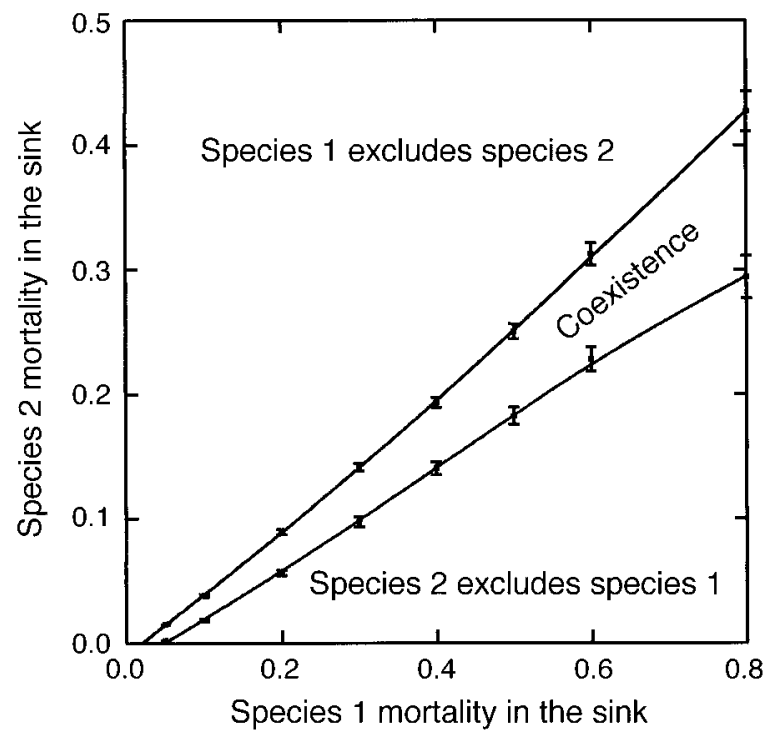

FIG. 2. With decreased competitive advantage of species 1 in the source $\left(\alpha_{12}=0.95, \alpha_{21}=1.05\right)$, the competitive inferior may exclude the competitive dominant when its advantage in the sink is large.

method for determining extinction is arbitrary, but highly repeatable between simulations (see error bars in Fig. 1 and 2). Varying the threshold density for extinction, the time window over which this density was averaged, or inspecting trends in long-term population time series all gave very similar results (K. Schmidt, unpublished data).

In model 2, we examined community structure and patterns of coexistence at varying levels of temporal stochasticity. Initial parameter values were chosen to result in stable habitat partitioning in the absence of temporal variability. We then increased environmental stochasticity (varying $\sigma$ between 0 to 0.60 ), solved for the ESS following the methods indicated above, and examined domains of parameter space permitting species coexistence.

\section{REsults}

Trade-off between competitive ability in the source and mortality in the sink yields coexistence...

With sufficient temporal stochasticity in a source habitat, use of a sink habitat becomes adaptive as a bet-hedging strategy (Holt 1997). Figure 1 shows domains of exclusion and coexistence when species 1 has a moderate competitive advantage in the source, and each species is at its respective ESS value for $p$. In the absence of temporal variability, species 1 is restricted to the source habitat where it excludes species 2 . Despite temporal variability and coupling with a sink habitat, the dominant competitor in the source (species 1) continues to excludes the inferior competitor unless there is a sufficient disparity between species' mortality rates in the sink habitat (Fig. 1). Increasing species 1's 
mortality in the sink increases the parameter space in which species 2 can coexist, but only up to a limit. The reason is that increasing species 1's mortality in the sink selects for higher values of $p$, until species 1 occupies the source habitat exclusively. Further increases to its sink mortality have no effect upon either species because species 1 no longer experiences the increased sink mortality. Higher intrinsic growth rates in the source magnifies the response of populations to temporal heterogeneity in $K$, which in turn increases the region of coexistence (Fig. 1) Similar effects arise with increased $\sigma$ (not shown).

Predictions from the Lotka-Volterra model may reverse under temporal stochasticity. When the species are nearly equal competitors, the competitive inferior in the source can exclude the dominant when its advantage in the sink is large (Fig. 2). It should be noted that for fixed values of $p$, coupling between a source and a sink habitat can lead to stable coexistence or reversed competitive domains even without temporal variability (K. Schmidt, unpublished results). However, in stable environments these ecologically feasible states will be evolutionarily transient if $p$ is free to evolve. Temporal variability in the source makes the ability to use a sink adaptively relevant, and can thus modify competitive interactions.

These results highlight a role that sink habitats may play in species coexistence, particularly in variable environments. In the first scenario, species 1's persistence at the landscape level is uninfluenced by the presence of the sink. However, species 2's persistence at both the local scale (source habitat) and at the landscape scale is critically tied to the sink habitat. The sink habitat acts as a site of temporal storage (Chesson and Rosenzweig 1991) for population recruitment resulting from good years in the source. Remove the sink, and the source population for species 2 also becomes extinct through competitive exclusion.

In the second scenario (reduced competitive dominance of species 1 ), even species 1 's persistence at the landscape level is influenced by the presence of the sink. Species 2 can exclude species 1 globally, despite being inferior in the habitat where competition is actually experienced. However, this result is contingent upon the sink habitat, and the presence of temporal variability in the source. Remove the sink or stabilize the source, and species 2 goes extinct (globally) because of competition with species 1 . Thus, species replacements may occur within habitats that are coupled through dispersal to additional habitats. Vincent et al. (1998) have shown that sink populations can be important for species persistence. Our results reinforce this conclusion. Given interspecific competition, sink habitats may control species composition in source habitats, permitting coexistence that otherwise would not be possible, or reversing competitive dominance. Temporal variability is necessary for these effects to

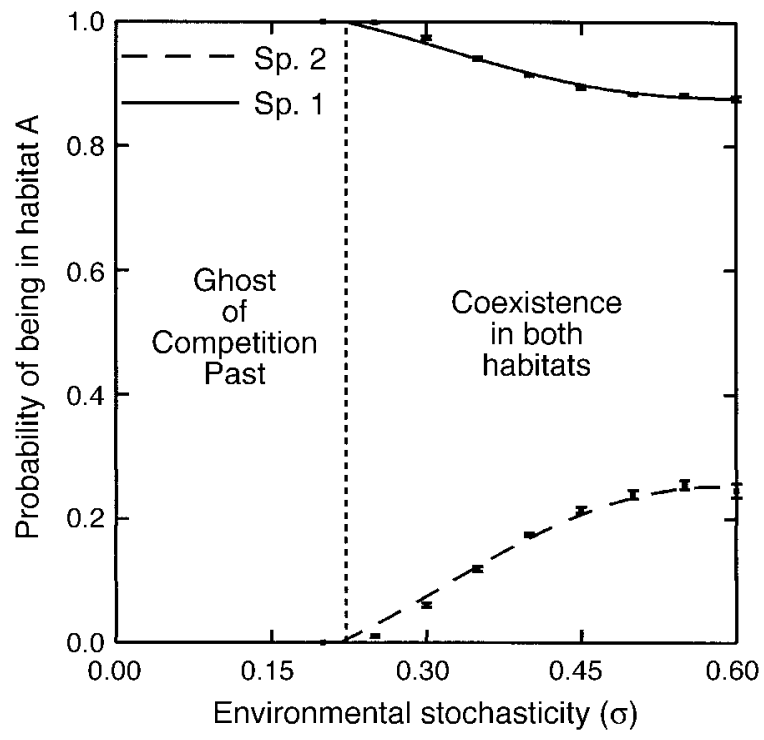

FIG. 3. The ESS ( $\pm 1 \mathrm{SE}$ ) solution (probability of being in habitat $\mathrm{A}$ ) as a function of environmental stochasticity, $\sigma$. With stable dynamics (i.e., no stochasticity), species 1 and 2 occupy separate habitats (A and B, respectively) at the ESS. Species evolve to be specialists and eliminate competitive interaction, "The Ghost of Competition Past." With temporal stochasticity, species evolve to use both habitats, and the Ghost vanishes. $\left(K_{1 A}=K_{2 B}=900 ; K_{1 B}=K_{2 A}=300 ; r_{1 A}=\right.$ $\left.r_{2 B}=1.2 ; r_{1 B}=r_{2 A}=0.1 ; \alpha_{12}=1.5 ; \alpha_{21}=1.2\right)$

be manifest, because such variability promotes the evolutionary stable use of sink habitats.

\section{... and the Ghost vanishes}

Without temporal variability, the initial conditions of model 2 result in habitat partitioning as the ESS, in which each species exclusively occupies a different habitat (Fig. 3). This habitat partitioning persists under low stochasticity. At moderate stochasticity $(\sigma=0.25)$, both species begin to use both habitats at the ESS (Fig. 3); environmental variability thus promotes habitat overlap of competing species with distinct habitat preferences. In our simulations, species 2 (the superior competitor) maintained a fairly steady total population density throughout the entire range of stochasticity (Fig. 4), whereas species 1's population density continued to decline as species 2 increasingly occupied both habitats at higher levels of stochasticity. Due to the competitive asymmetry, species 2 occupied habitat A to greater extent than species 1 did habitat B (Fig. 3).

\section{Breakdown of coexistence under temporal variability}

Coexistence at an ESS was not the only outcome that occurred under temporal variability. Another possible scenario was global extinction (Fig. 5) of one of the competitors. In this scenario, species 1 begins to utilize both habitats as temporal variability increases. The higher intrinsic growth rate of species 1 in its preferred habitat $\left(r_{1 A}=1.5\right)$ makes this species experience tem- 


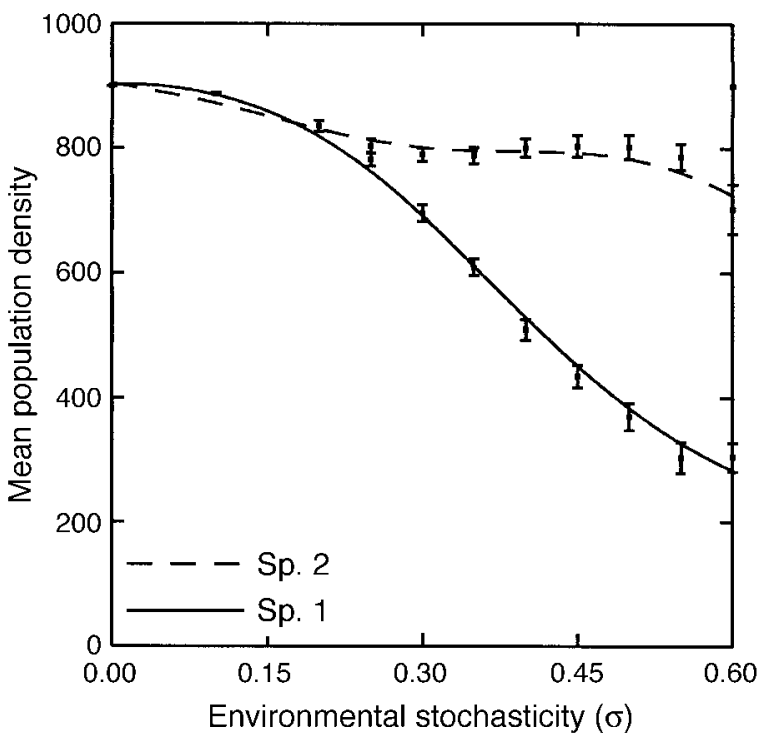

FIG. 4. Mean population density ( $\pm 1 \mathrm{SE}$ ) for the scenario depicted in Fig. 3. Mean density was calculated by averaging each species' total population size over the last 100 generations of the simulations. Species 2 is competitively dominant and maintains a fairly constant average density throughout the range of stochasticity, whereas the density of species 1 declines with increasing stochasticity.

poral stochasticity more strongly and so prompts the evolution of dispersal at lower environmental stochasticity than for species $2\left(r_{2 B}=1.0\right)$. The result is increased use of habitat $B$ by species 1 , which drives down the density of species 2 (Fig. 6), further inhibiting use of both habitats by species 2. At high levels of temporal variability, species 2 should use both habitats as the ESS; however, it cannot persist ecologically (Fig. $6)$.

\section{DISCUSSION}

Traditional optimal habitat selection is in effect predicated on being in the right place in a spatially heterogeneous world. Optimal habitat selection under temporal variability is subtly different, "ecological instability favors the evolution of genotypes with behavioral flexibility to avoid being in the wrong place at the wrong time" (Fryxell 1997:698). Dispersal to sink habitats can be adaptive as a bet-hedging strategy even in the absence of a despotic distribution; a nonzero probability of using sink habitat can be evolutionarily advantageous (Holt 1997). Here we have shown that this evolutionary impact of temporal variation on habitat use can either promote species coexistence or lead to competitive exclusion.

First, the mechanism of coexistence permitted by hard sinks highlights how individual behavior links local with regional scales of interactions. In our first model, coexistence requires a trade-off between competitive ability in the source and mortality in the sink. Such trade-offs may be common in mobile organisms (e.g.,

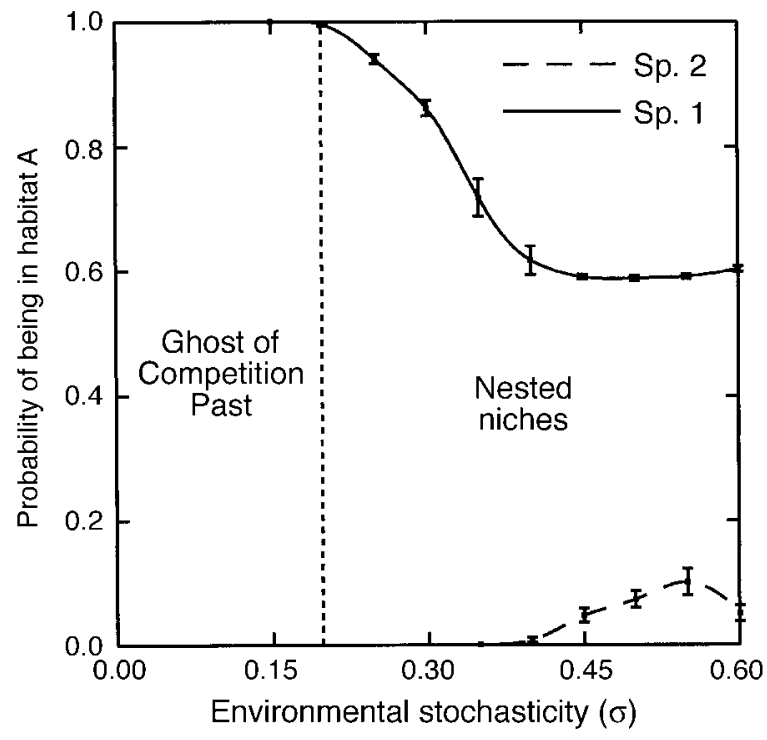

FIG. 5. The evolutionary stable strategy (ESS) solution (probability of being in habitat $\mathrm{A} ; \pm 1 \mathrm{SE}$ ) as a function of environmental stochasticity. With stable dynamics (i.e., no stochasticity), species 1 and 2 occupy separate habitats (A and B, respectively) at the ESS. With temporal stochasticity, species 1 evolves to be a habitat generalist while species 2 remains a habitat specialist until finally becoming extinct. This type of community organization is called nested niches. $\left(K_{1 A}=1000 ; K_{1 B}=500 ; K_{2 A}=300 ; K_{2 B}=800 ; r_{1 A}=1.5 ;\right.$ $\left.r_{1 B}=0.1 ; r_{2 A}=0.1 r_{2 B}=1.0 ; \alpha_{12}=1.5 ; \alpha_{21}=1.2\right)$

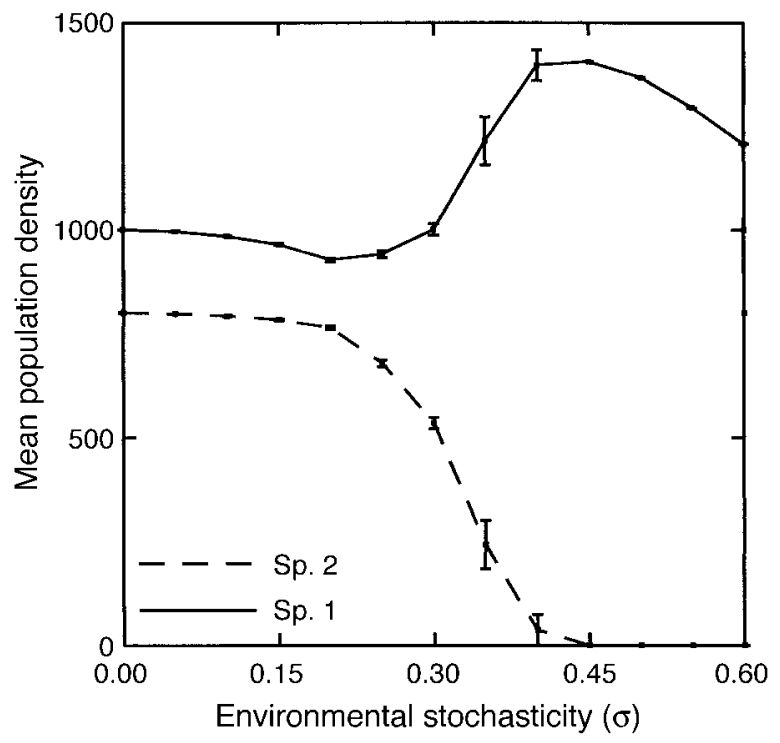

FIG. 6. Mean population density ( $\pm 1 \mathrm{SE}$ ) for the scenario depicted in Fig. 5. Average densities are relatively unaffected by temporal variability as long as both species specialize on their preferred habitat. After species 1 evolves to use both habitats, its average density initially increases with further increases in temporal variability. However, the average density of species 2 , restricted to habitat $\mathrm{B}$, decreases toward extinction as variability increases. 
Werner and Hall 1988, McPeek 1990, Werner and Anholt 1993, Grill and Juliano 1996). For instance, mobility is often associated with greater foraging efficiency and/or faster growth rates that enhance competitive ability (Grill and Juliano 1996). At the same time, mortality from predation is also positively related to mobility (Werner and Hall 1988, Werner and Anholt 1993).

Alternatively, consider habitats in time where seed germination in a good year represents a potential source and the seed bank a sink. Large seeds have a competitive advantage over smaller seeds upon germination (Sork 1987, Westoby et al. 1992), but may suffer higher predation through size-selective foraging by seed predators (e.g., Kelrick et al. 1986, Podolsky and Price 1990) and thus are poor candidates for long-term survival in seed banks (Thompson and Grime 1979). Interestingly, most of the variation in seed size within a species is due to variation within individual parental plants (Michaels et al. 1988) that appear to hedge their bets between the conflicting responses to competition and predation. This last example illustrates that comparable phenomena to those considered here have been a recurring theme in the literature on life history effects on coexistence (Ellner 1987, Chesson and Huntly 1988, Hairston et al. 1997).

Second, we have also shown that temporal stochasticity that can create a novel mechanism of coexistence can, in other circumstances, conversely modify or eliminate traditional mechanisms of coexistence based on habitat selection. Habitat partitioning breaks down because temporal variability leads to species using a broader range of habitat types. The stable form of coexistence based on habitat selection in the absence of stochasticity disappears, with one species potentially eliminating the other at high levels of stochasticity. Recent analyses (Chesson and Huntly 1997) have suggested that population fluctuations (driven biotically or abiotically) and environmental harshness do not necessarily modify the conditions of competitive coexistence for broad classes of ecological models with linear per capita dynamics. However, our models combine temporal and spatial heterogeneity, leading to phenomena absent when considering either alone. Temporal fluctuations need not diminish the impacts of competition in determining community patterns of species distribution (e.g., Wiens 1977, Huston 1979); instead, we suggest that fluctuations may actually increase competition by reducing the likelihood of habitat partitioning, and so lead to competitive exclusion via flow of individuals across landscapes.

A further evolutionary outcome may also make longterm competitive coexistence more difficult in spatially heterogeneous environments. As indicated by Holt (1997) and Rosenzweig (1987), temporal variability or unstable population dynamics favors the utilization of more habitats. Species are then tested against the selective forces averaged over those habitats they inhabit, such that species are more likely to become habitat generalists (Holt 1996) reducing the likelihood of habitat specialization as a mechanism of coexistence.

Finally, we can draw some comparisons between our results and metapopulation analyses. In a simple twopatch, two-species metapopulation, we were able to obtain varying patterns of local and regional persistence and coexistence of two competitors. These outcomes arose not as the result of a balance between the extinction and colonization of populations among habitat patches, the focus of metapopulation theory (Hanski 1998), but as a consequence of adaptive, individual behavior. Dispersal or movement between habitat patches in our models is a regional phenomenon that arose out of a consideration of local population dynamics. Cautionary conclusions for metapopulation theory that may be drawn are: (1) local population dynamics may be critical to establishing coexistence within a metapopulation; (2) coexistence may require or may break down under sufficient temporal stochasticity; and (3) coexistence may be evolutionarily unstable under varying regimes of temporal stochasticity.

\section{Caveats and future directions}

In our simulations, we assumed both species experienced the same level of environmental stochasticity in any given year and between habitats (model 2 ). This was necessary to investigate whether a trade-off between competitive ability in a source and survival in a sink could influence coexistence between the two competitors. We parameterized the model community such that the dominant competitor always excluded the inferior competitor from the source habitat (model 1) or its preferred habitat (model 2) in the absence of temporal variability. Covariation in temporal stochasticity insured that the requirement for exclusion held for any given year. Under a scenario in which species experience levels of environmental stochasticity independently or semi-independently of one another, coexistence may occur more often than our models indicate, or, as noted by Hutchinson (1961), more generations may be required to reach competitive exclusion. This occurs because in some years the requirements for competitive exclusion in the absence of stochasticity (Eq. 5) may not be met or may be reversed. Relaxing the assumption of covariation in temporal stochasticity between species or incorporating other patterns of temporal stochasticity (e.g., good and bad years come in runs) may influence patterns of coexistence in interesting ways, but goes beyond the scope of the present study.

Our models assume that organisms either cannot assess and/or cannot respond to changes in habitat quality from year to year. Organisms do not move into the better habitat or distribute themselves between habitats in an ideal free manner. Rather, dispersal is ecologically fixed in the form of the parameter $p$. When organisms possess the behavioral flexibility to adjust to variability 
in habitat quality within years, our results may no longer hold. However, in some scenarios, the behavior of our model organisms may be better approximated. For instance, songbirds, especially migratory species, redistribute themselves across a landscape after spring and fall migrations. Settling decisions of individuals may be far from perfect because at the time of settling, resources or predator densities cannot be accurately assessed (Orians 1980), or predator density, when it can be assessed, may be a poor indicator of expected breeding success (Schmidt 1999; K. Schmidt and J. Goheen, unpublished manuscript). Habitats often have predictably higher quality, on average, than others (wintering habitat: Marra et al. 1998; breeding habitat: Holmes et al. 1996, Morse and Robinson 1999). Birds may therefore disperse between habitat types between and/or within years, biasing settlement toward higher quality habitats. In these regards, some migratory birds may fit the assumptions of our models, suggesting spatial heterogeneity and temporally variability may influence their community structure in ways we have explored.

Variability in space and time is pervasive throughout ecological communities, and thus we might expect the effects we have illustrated to be fairly common. Nonetheless, finding explicit examples may prove very difficult. For instance, the storage effect first proposed by Chesson and Warner (1981) has seen very little empirical documentation other than the accumulation of examples that show conditions for its operation are quite common in nature (Hairston et al. 1997). Organisms survive bad times by storing the productivity of good times. We have built upon this idea the concept that storage in time may be spatially explicit and may influence community organization beyond the effects of temporal storage alone.

In conclusion, temporal stochasticity leading to dispersal between habitats, acts as a bet-hedging tool in a source-sink system, creating new forms of trade-offs and hence new mechanisms of coexistence. However, the same stochasticity that encourages increased habitat breadth may increase the likelihood of intense competition and the potential for competitive exclusion. Because most species inhabit a world which is both spatially heterogeneous and temporally variable, we suggest that our results are likely important to the determination of coexistence and community structure in a broad range of natural communities of mobile organisms.

\section{ACKNOWLEDGMENTS}

We thank students of the Fall 1996 Game Theory class at the University of Illinois at Chicago and Doug Morris for many insightful discussions and comments on the nature of habitat selection. F. Adler and two anonymous reviewers provided useful comments to an earlier version. This paper is a contribution to the program of the Institute of Ecosystem Studies. Financial support during preparation was provided by the National Science Foundation (DEB 9615414).

\section{Literature Cited}

Brew, J. S. 1982. Niche shift and the minimization of competition. Theoretical Population Biology 22:367-381.

Brown, J. S. 1990. Habitat selection as an evolutionary game. Evolution 44:732-746.

Brown, J. S. 1996. Coevolution of community organization in three habitats. Oikos 75:193-206.

Brown, J. S. 1997. Game theory and habitat selection. Pages 188-220 in L. A. Dugatkin and H. K. Reeve, editors. Game theory and animal behavior. Oxford University Press, Oxford, UK.

Chesson, P., and N. Huntly. 1988. Community consequences of life-history traits in a variable environment. Annales Zoologi Fennici. 25:5-16.

Chesson, P., and N. Huntly. 1997. The roles of harsh and fluctuating conditions in the dynamics of ecological communities. American Naturalist. 150:519-553.

Chesson, P., and M. L. Rosenzweig. 1991. Behavior, heterogeneity, and the dynamics of interacting species. Ecology 72:1187-1195.

Chesson, P. L., and R. R. Warner. 1981. Environmental variability promotes coexistence in lottery competitive systems. American Naturalist 117:923-943.

Connell, J. H., and W. P. Sousa. 1983. On the evidence needed to judge ecological stability or persistence. American Naturalist. 121:789-824.

Doebeli, M., and G. D. Ruxton. 1997. Evolution of dispersal rates in metapopulation models: branching and cyclic dynamics in phenotype space. Evolution 51:1730-1741.

Ellner, S. 1987. Alternate plant life history strategies and coexistence in randomly varying environments. Vegetatio 69: 199-208.

Fryxell, J. M. 1997. Evolutionary dynamics of habitat use. Evolutionary Ecology 11:687-701.

Fryxell, J. M., B. F. Falls, E. A. Falls, and R. J. Brooks. 1998. Long-term dynamics of small mammal populations in Ontario. Ecology 79:213-225.

Grill, C. P., and S. A. Juliano. 1996. Predicting species interactions based on behavior: predation and competition in container-dwelling mosquitoes. Journal of Animal Ecology 65:63-76.

Hairston, N. G., Jr., S. Ellner, and C. M. Kearns. 1997. Overlapping generations: the storage effect and the maintenance of biotic diversity. Pages 109-145 in O. E. Rhodes, Jr, R. K. Chesser, and M. H. Smith, editors. Population dynamics in ecological space and time. University of Chicago Press, Chicago, Illinois, USA.

Hanski, I. 1998. Metapopulation dynamics. Nature 396:4149.

Holmes, R. T., P. P. Marra, and T. W. Sherry. 1996. Habitatspecific demography of breeding Black-throated Blue Warblers (Dendroica caerulescens): implications for population dynamics. Journal of Animal Ecology 65:183-195.

Holt, R. D. 1985. Population dynamics in two-patch environments: some anomalous consequences of an optimal habitat distribution. Theoretical Population Biology 28: 181-208.

Holt, R. D. 1996. Adaptive evolution in source-sink environments: direct and indirect effects of density-dependence on niche evolution. Oikos 75:182-192.

Holt, R. D. 1997. On the evolutionary stability of sink populations. Evolutionary Ecology 11:723-731.

Holt, R. D., and M. A. McPeek. 1996. Chaotic population dynamics favors the evolution of dispersal. American Naturalist 148:709-718.

Huston, M. 1979. A general hypothesis of species diversity. American Naturalist 113:81-101.

Hutchinson, G. E. 1961. The paradox of the plankton. American Naturalist 95:137-146.

Keddy, P. A. 1981. Experimental demography of the sand- 
dune annual, Cakile edentula, growing along an environmental gradient in Nova Scotia. Journal of Ecology 69: 615-630.

Kelrick, M. I., J. A. MacMahon, R. R. Parmenter, and D. V. Sisson. 1986. Native seed preferences of shrub-steppe rodents, birds, and ants: the relationship of seed attributes and seed use. Oecologia 68:327-337.

MacArthur, R. H. 1972. Geographical Ecology. Harper and Row, New York, New York, USA.

Marra, P. P., K. A. Hobson, and R. T. Holmes. 1998. Linking winter and summer events in a migratory bird by using stable-carbon isotopes. Science 282:1884-1886.

McPeek, M. A. 1990. Behavioral differences between Enallagma species (Odonata) influencing differential vulnerability to predators. Ecology 71:1714-1726.

Michaels, H. J., B. Benner, A. P. Hartgerink, T. D. Lee, S. Rice, M. F. Willson, and R. I. Bertin. 1988. Seed size variation: magnitude, distribution, and ecological correlates. Evolutionary Ecology 2:157-166.

Morris, D. W. 1991. On the evolutionary stable dispersal to sink habitats. American Naturalist 137:907-911.

Morse, S. F, and S. K. Robinson. 1999. Nesting success of a Neotropical migrant in a multiple-use forested landscape. Conservation Biology 13:327-337.

Orians, G. H. 1980. Some adaptations of marsh-nesting blackbirds. Princeton University Press, Princeton, New Jersey, USA.

Parker, G. A., and W. J. Sutherland. 1986. Ideal free distributions when individuals differ in competitive abilities: Phenotype-limited ideal free models. Animal Behavior 34: 222-1242.

Podolsky, R. H., and M. V. Price. 1990. Patch use by Dipodomys deserti (Rodentia: Heteromyidae): profitability, preference, and depletion dynamics. Oecologia 83:83-90.

Pulliam, H. R. 1988. Sources, sinks, and population regulation. American Naturalist. 132:652-661.

Pulliam, H. R., and B. J. Danielson. 1991. Sources, sinks, and habitat selection: a landscape perspective on population dynamics. American Naturalist 137:S50-S66.
Rosenzweig, M. L. 1981. A theory of habitat selection. Ecology 62:327-335.

Rosenzweig, M. L. 1987. Habitat selection as a source of biological diversity. Evolutionary Ecology 1:315-330.

Rosenzweig, M. L. 1991. Habitat selection and population interactions: the search for mechanisms. American Naturalist 137:S5-S28.

Schmidt, K. A. 1999. Foraging theory as a conceptual framework for studying nest predation. Oikos 85:151-160.

Shmida, A., S. Ellner. 1984. Coexistence of plant species with similar niches. Vegetatio 58:29-55.

Sork, V. L. 1987. Effects of predation and light on seedling establishment in Gustavia superba. Ecology 68:1341-1350.

Thompson, K., and J. P. Grime. 1979. Seasonal variation in the seed banks of herbaceous species in ten contrasting habitats. Journal of Ecology 67:893-921.

Vincent, A., A. Jansen, and J. Yoshimura. 1998. Populations can persist in an environment consisting of sink habitats only. Proceedings of the National Academy of Science 95: 3696-3698.

Vincent, T. L., and J. S. Brown. 1984. Stability in an evolutionary game. Theoretical Population Biology 26:408427.

Watkinson, A. R., and W. J. Sutherland. 1995. Sources, sinks, and pseudo-sinks. Journal of Animal Ecology 64:126-130.

Werner, E. E., and B. R. Anholt. 1993. Ecological consequences of the trade-off between growth and mortality rates mediated by foraging activity. American Naturalist 142 : 242-272.

Werner, E. E., and D. J. Hall. 1988. Ontogenetic habitat shifts in bluegill: the foraging rate-predation risk trade-off. Ecology 69:1352-1366.

Westoby, M., E. Jurado, and M. Leishman. 1992. Comparative evolutionary ecology of seed size. Trends in Evolution and Ecology 7:368-372.

Wiens, J. A. 1977. On competition and variable environments. American Scientist: 65:590-597.

Wilson, D. S., and J. Yoshimura. 1994. On the existence of specialists and generalists. American Naturalist 144:692707 